\title{
Bushfire propagation speed: Combining the effects of wind and slope
}

\author{
$\underline{\text { S. Berres }}^{a}$ (iD) and N. Cárcamo ${ }^{b}$ \\ a Universidad del Bío-Bío, Chillán, Chile \\ ${ }^{\mathrm{b}}$ Universidad Católica del Maule, Talca, Chile \\ Email: Stefan.Berres@gmail.com
}

\begin{abstract}
In this contribution the effect of slope inclination on the bushfire propagation speed is studied. The slope effect is estimated in an inverse problem setup. As observation data, real fire scenarios are given in terms of maximum fire expansions. With the knowledge of the initial ignition location, the front perimeters can be determined in any direction. The direct problem is formulated as a mathematical model of surface bushfire. It is expressed in a functional form, where the front propagation speed depends on wind and slope. The fuel material as predominant factor is given as a constant, i.e. no heterogeneous combustible effects are considered, and the effects of humidity are neglected. The observation data are given as radial front propagation perimeter. As model and data are two dimensional, the wind impact is decomposed in speed and direction; the speed is the maximal speed in wind direction, such that in the orthogonal direction to the wind there is zero speed and negative maximum speed in the opposed wind direction. In the model, the speed parameters are fixed for the overall domain, whereas the inclination is known from topographical data.
\end{abstract}

The parameter identification problem is formulated as a nonlinear optimization problem, where the distance of the parametric model to the data is minimized by the optimal parameter set. The observation data give the distance reached by the propagation front. Though the radial perimeter data are two dimensional, covering all 360 degree directions, the model decouples the directions, establishing for each direction a one dimensional model. In this model simplification it is assumed that the fire spreads in each direction separately, without cross-directional interference. The mathematical model describes the propagated pathway simultaneously for each direction by a differential equation, where the change of position in time is given by the velocity model, that expresses the velocity in terms of wind and slope. In general, the model solution at given time points can be compared with the measured advance of the propagation front of a particular fire. The measurement data of final bushfire perimeters is provided by the National Forest Corporation of Chile (CONAF). The used experimental data is implemented manually from a shapefile type file using Google Earth tools. The methodology of solving the inverse optimization problem is implemented computationally on the Matlab/Octave, with plans to migrate to Python.

The sensitivity analysis gives only a weak validation of the slope dependence. As a conclusion, more data than the final perimeter data are required. In standard situations, satellite images have been available only once a day, and firefighters are not yet by default equipped with GPS sensors that might enable local firefront tracking. The expectation is that in the context of the big data paradigm, i.e. within the omnipresence of ubiq-uitious computing, a denser data coverage is going to be available, that might be coordinated by corresponding projects. Regarding the model, certainly the simplification of a two-dimensional scenario by simultaneous one-dimensional models is restrictive, especially in the perspective that the interaction with meteorological aspects requires a three-dimensional model, that even might be enhanced by multiple scales. Yet, the dif-ferent model types might contribute to the discussion in the context of the availability of data and different comprehension levels of involved users or stakeholders. Thus, the presented model is going to be still valid for didactic purposes and as an intermediate parameter identification approach, that goes fine within an open modelling methodology.

Keywords: Bushfire, propagation perimeter, parameter estimation, sensitivity analysis 


\section{INTRODUCTION}

Bushfire propagation models have been formulated to systematize the collection of necessary and useful prior information that helps to get prepared to attacking an outbreaking fire. The reconsideration of bushfire models is a more academic exercise that only partly gets involved in the complex real phenomenon; but it can contribute to the systematization efforts and contribute to the prediction potential. If the developed models are well designed then they can find their way into an operational or experimental use as soon as follow-up data driven studies are carried out. Particular studies for specific fires are those that have yielded the best results due to the augmented information that they contain such as the soil characteristics, combustible material, humidity or the surface slope. The management of real parameters is crucial to characterize the physical phenomenon, as shown by the models mentioned in (Dumond 2009, Alexander \& Cruz 2013, Cassagne et al. 2011).

The original motivation of this work was to experiment with descriptions of the behavior of the bushfire propagation by robust and simple mathematical models in terms of differential equations, with the perspective to plug these models into prediction algorithms. A hypothesis of this study is that two-dimensional surface models can describe the fire front propagation effectively. The more versatile and numerically robust method built on the formulation of the two-dimensional phenomenon is done by Hamilton-Jacobi equations as developed within the level set method (Hilton et al. 2015, Mallet et al. 2009, Ghisu et al. 2014, Rehm \& McDermott 2009 Osher \& Sethian 1988, Sethian 1996). However, frequently the general data access is relatively scarce, representing an obstacle when trying to fit and validate this model in real contexts; moreover, in order to employ advanced model types, a crucial question is the basic bushfire modeling issue, what propagation rate model should be employed. Thus, there is a need for assessing the contextualized propagation model in a simplified experimental setting. Whereas the role of fuel type and wind is relatively well documented, one major question is the effect of the slope of the terrain surface. This contribution assesses the effect of surface slopes by trying to adjust a mathematical model that contains information of the slope to experimental data in form of final bushfire propagations.

Impact of topographic factors. Topographic factors refer to the effect of the slope of the terrain on how the energy is transferred to the fuel and the atmosphere in the burning process. A general implication on modeling is for example the fact that the upward slope is burned faster than the downward slope (Sullivan 2007), as heat is transmitted by convection and radiation more intensely in uphill direction. Finney (Finney 1998) and other authors (Viegas 2004, Beer 1993) propose the assignment of vectors to the combined wind-slope effect, that causes variations of the propagation direction for non-combined effects. There is a powerful consequence of the joint action of wind and slope that contributes to the quickness in which the ignition temperature is reached in the direction where the heat is transmitted. If the wind is blowing up the slope, then the propagation speed increases, and if the wind is blowing down the slope, the propagation direction can be estimated with the wind-slope vector assignment.

\section{MATHEMATICAL MODEL}

For the quantitative model we set up a reference system in polar coordinates $(x(\phi), \phi)$ where the origin corresponds to the ignition point, such that $x(\phi)$ is the propagation radius that depends on the angle $\phi$ of the corresponding wind direction. In the model it is assumed that in each fire spread direction there is an autonomous propagation without interference between different directions. The start of the bushfire is placed in the origin of the reference system and in each direction there is a line of propagation advance. See Figure 1 for the data expressed in terms of the polar reference coordinates. These data are used to adjust the decoupled dimensional propagation models.

If in the wind direction $\phi^{\star}$ the wind velocity is $U^{\star}$ then the wind velocity in direction $\phi$ can be expressed as

$$
U=\cos \left(\phi-\phi^{\star}\right) U^{\star},
$$

where $\cos \left(\phi-\phi^{\star}\right)$ corresponds to the scalar product between the direction of the wind and the considered propagation direction, giving 0 if the directions are orthogonal, and -1 if opposed.

Different velocity models are proposed in order to distinguish the effect of parameters onto the final bushfire propagation, and to graphically compare the different effects of a linear or exponential dependence, as the two main model types presented in the literature (Weise \& Biging 1994).

Two dimensional propagation model. Bushfire propagation models generally have a linear or exponential formulation, where an exponential formulation allows us to express the speed-up effect for accelerating wind. 


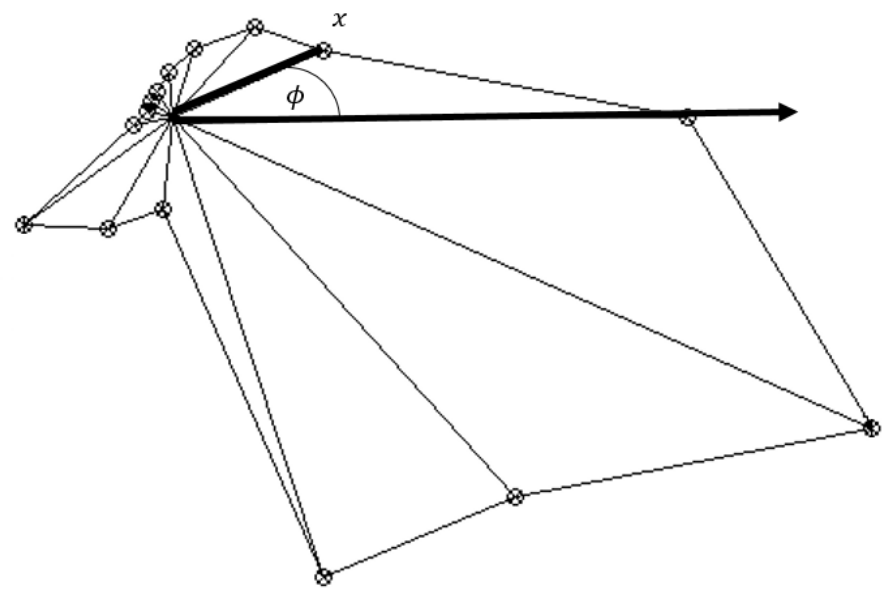

Figure 1. Representation of final perimeter of bushfire within reference system, that indicates the propagation in each direction. The origin corresponds to the ignition point.

At a low wind velocity there is a retarding effect, whereas at higher wind velocities the effect gets accelerated. A reviewer commented that this is likely to depend on the intensity of the fire, e.g. as described by the Byram convective number.

As a reference model, the bushfire propagation speed

$$
R(U, \theta, x, \phi)=a e^{b U \cos \left(\phi-\phi^{\star}\right)+c \theta(x, \phi)}
$$

depends on the maximum wind velocity $U$, the wind direction $\phi$, the directional surface slope $\theta$, and parameters $a, b, c$ that are to adjust to the data.

In the reference model (2) the slope effect is aggregated to the flat surface model by the multiplication of an extra function, as proposed in Noble et al. (1980), see Cruz et al. (2014), p. 8 and p. 34; as a rational of this choice, the functional behaviour of exponential functions have reasonable qualitative properties to start with, reducing to a constant effect at zero-wind and zero-slope, and extending to an upspeeding behaviour for increasing variable values. As basic property of exponential functions, their multiplication, as a multiple composition of two of them, turns into an additive composition within one exponential function; this behaviour is in contrast to Rothermel type models as an alternative, which propose an additive composition of different effects.

The surface slope $\theta$ depends on the position and is pre-calculated based on topographic data. Based on local elevation data, in each direction the heights are interpolated by a cubic spline, i.e. a piecewise cubic polynomial. In absence of wind and slope, i.e. when $U=0$ and $\theta=0$, the corresponding effect disappears since $e^{0}=1$, such that the bushfire propagation speed becomes constant.

The evolution of the bushfire is modeled as a differential equation

$$
\frac{d x}{d t}=R(U, \theta(x)), \quad x(0)=0
$$

where $U$ is calculated from equation (1) and the slope $\theta=\theta(x)$ is position dependent. The integration of the differential equation ( 3 ) is done numerically, delivering the reached positions in each different direction.

Dealing with different directions

$$
\phi_{j}, \quad j \in \mathcal{J}=\{1, \ldots, J\}
$$

the simultaneous 1D model becomes

$$
\frac{d x_{j}}{d t}=R\left(U_{\phi_{j}}, \theta_{j}\left(x_{j}\right)\right), \quad U_{\phi_{j}}=\cos \left(\phi_{j}-\phi^{\star}\right) U^{\star} .
$$

Solving equation (4), the value $x_{j}(T)$ corresponds to the fire advance at time $T$ in direction $\phi_{j}$, which in turn is compared to experimental data in order to obtain the optimal parameter set; here, the value $x_{j}(T)$ is just the numerically calculated value in the direction $\phi_{j}$ corresponding to the end of the time interval $[0, T]$. 
Stefan Berres and Noemí Cárcamo, Bushfire propagation speed: Combining the effects of wind and slope

Inverse problem. The parameter set related to a specific experiment can be expressed as

$$
P=\left(\phi^{\star}, U^{\star}, \theta \cdot(\cdot)\right) .
$$

If there are several experiments that are identified by indices $i \in\{1, \ldots, I\}$, then for each experiment $V_{i}$ we want to find an optimal parameter set $P_{i}$ that minimizes

$$
\min _{P_{i}} f\left(P_{i}\right), \quad f\left(P_{i}\right)=\sum_{j=1, \ldots, J} \frac{\left|x_{i}^{j}\left(P_{i} ; T\right)-\hat{X}_{i}^{j}\right|^{2}}{\left(\hat{X}_{i}^{j}\right)^{2}},
$$

the difference between the experimental propagation data and the solution of the differential equation. Here, $x_{i}^{j}\left(P_{i} ; T\right)$ is the solution with the parameter set $P_{i}$ and $\hat{X}_{i}^{j}$ the corresponding real data. In (6), the relative errors are summed up, where $J$ corresponds to the number of direction, i.e. the advance line for each respective experiment, see Figure (1).

The challenge is to identify optimal parameters that, when incorporated into the equation (3) together with solving the direct problem, show a final spread perimeter of the forest fire similar to the spread perimeter of the study areas. For a given number of lines we have the following angles:

$$
\phi_{i j}=j \Delta \phi=\frac{j 2 \pi}{J}, \quad j=1, \ldots, J .
$$

With given parameters, the direct problem is solved by finding the maximum distance that the fire reaches in a given time. With that information, the cost function (6) can be optimized to determine the best parameters that provide a minimum error with respect to the data.

From the shapefile archive the elevation profiles of the terrain in different directions can be obtained. Given the set of selected points

$$
\hat{X}_{i j}=\left(x_{i j 1}, x_{i j 2}, \ldots, x_{i j K}\right), \quad x_{i j K}=\hat{X}_{i}^{j}, \quad \hat{Z}_{i j}=\left(z_{i j 1}, z_{i j 2}, \ldots, z_{i j K}\right),
$$

the elevation profile can be represented as a cubic spline, i.e. a piecewise cubic interpolation

$$
C_{i j k}(x)=s_{i j k, 0}+s_{i j k, 1}\left(x-x_{i j k}\right)+s_{i j k, 2}\left(x-x_{i j k}\right)^{2}+s_{i j k, 3}\left(x-x_{i j k}\right)^{3}
$$

with $k$ pieces, and polynomial parameters $\left(s_{j k, c}\right)_{c=0}^{3}$. The same way the value of the slopes at each position are obtained as

$$
\theta(x)=\frac{d C_{i j k}}{d x}=s_{i j k, 1}+2 s_{i j k, 2}\left(x-x_{i j k}\right)+3 s_{i j k, 3}\left(x-x_{i j k}\right)^{2} .
$$

By interpolation, the slope $\theta(x)$ corresponding to the sets of positions $\left(\hat{X}_{i j}, \hat{Z}_{i j}\right)$ is recovered. The distances and slopes do not represent a discrete number of data, but a continuity of interpolated data. The result of the interpolation is incorporated into the governing differential equation.

\section{METHODS: COMPUTATIONAL IMPLEMENTATION}

To solve the problem under study, computational codes are created and computer simulations performed. The codes are implemented in Matlab, with the perspective to migrate to Octave or Python. First, a function is created that contains the equation (2); this function is incorporated into a script that solves the direct problem. In this script the initial speed and the wind direction can be set; the function that describes the slope is also incorporated. Then, a script is generated to solve the equation (3) for each studied model variant; this script in turn is called from another script that solves the inverse problem. This script for the solution of the inverse problem is on the highest level of the implementation.

The data provided by CONAF are in the form of maps of forest fires, where there is a record of the starting point of the fire and the final spread perimeter. With each of them, the advance and height data in the 15 lines of advance of the fire are recovered as shown in Figure 2; moreover, through the Google Earth elevation profiles tool, the advance in $\mathrm{X}$ and the $\mathrm{Z}$ height can be obtained for each direction. The number of 15 lines was an arbitrary design choice, as it happens to be some multiple of 5, in the perspective that 10 seemed too little and 20 too much from a heuristic perspective; in retrospect, 12 or 16 lines would appear more natural as they align to the cardinal directions; yet, $360=15 \cdot 24$ is still within a non-fractional calculus. 
Stefan Berres and Noemí Cárcamo, Bushfire propagation speed: Combining the effects of wind and slope

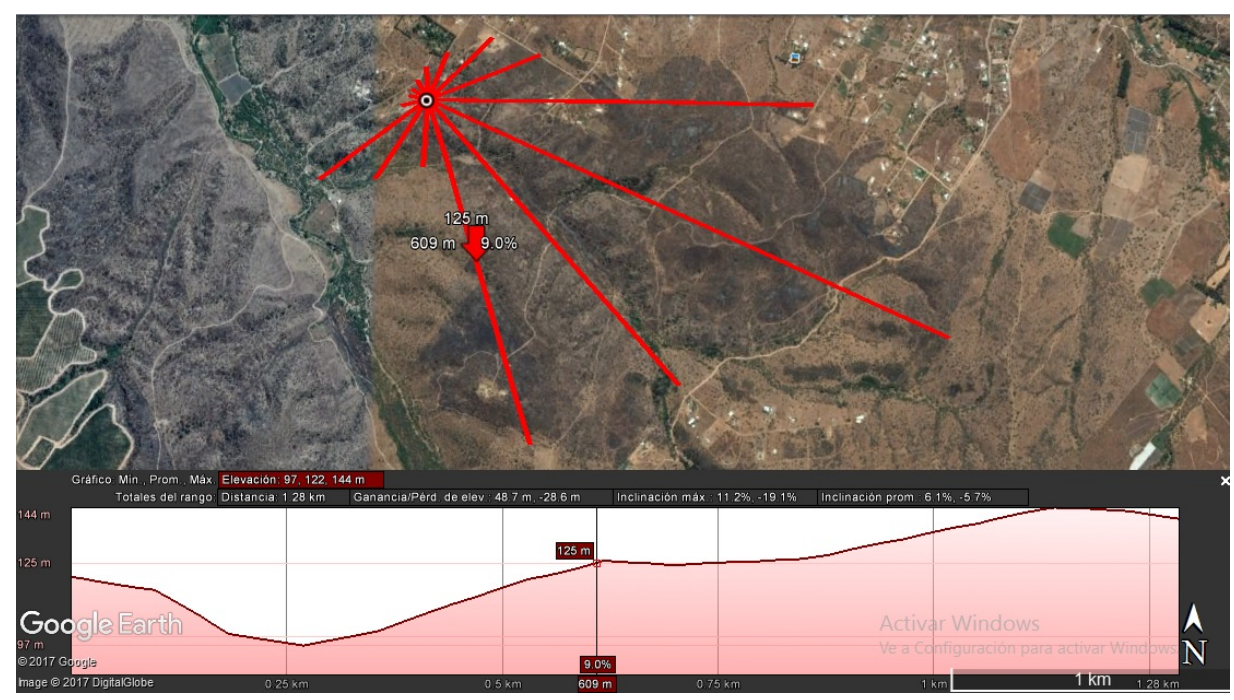

Figure 2. Lines in the bushfire advance directions of the event of Los Maitenes. Inset: Height profile in selected direction. Image taken from Google Earth.

(a)

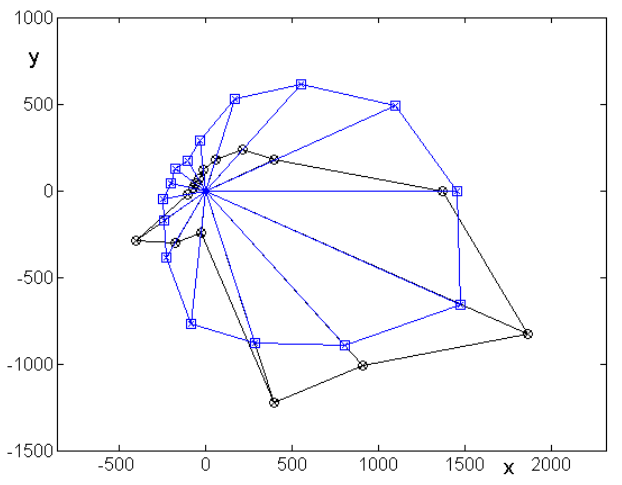

(c)

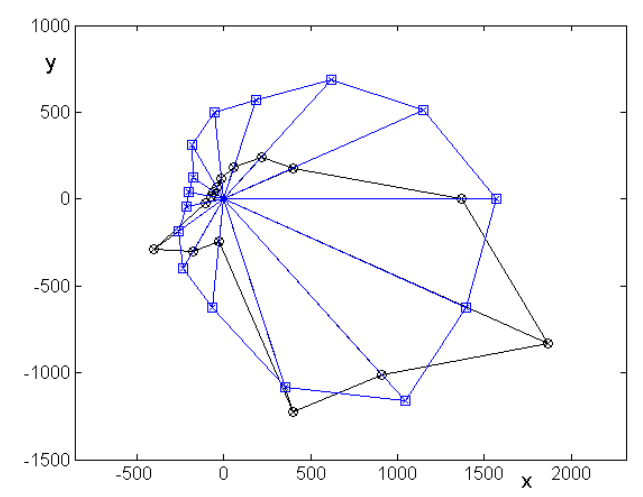

(b)

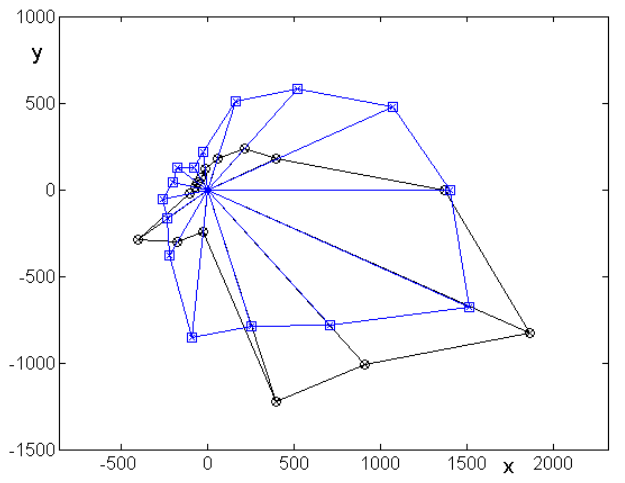

(d)

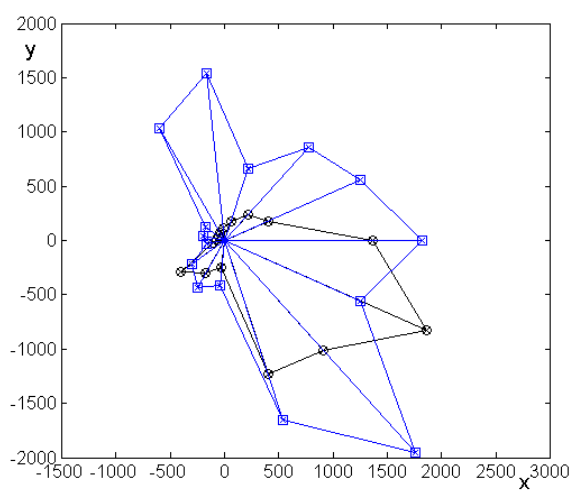

Figure 3. Variation of parameter $c$ in the Los Maitenes bushfire, with $a=5.6947, b=1.0230, \phi^{\star}=-0.3$; (a) $c=-1$ (b) $c=-2$ (c) $c=2$ (d) $c=5$. 


\section{RESULTS}

In Figure 3, the effect of the parameter $c$ in the Los Maitenes bushfire is demonstrated by visualizing solutions of the direct problem with fixed parameters $a, b, \phi^{\star}$, but varying values of $c$. Note that the parameter $c$ controls the effect of terrain slope. Low parameters of $c$ correspond to a small slope sensitivity generating homogeneous spread distances, that are biased towards the wind direction. In Figure 3 (a) there is an underestimation of the slope effect, in Figure 3 (d) an overestimation.

The difference between the predictions of model (2) and experimental data shown in Figure 3 can be explained by a combination of the following effects: (1) heterogeneities in terms of non-homogeneous fuel distributions that are not recorded in detail, (2) additional effects as moisture, or (3) the non-documented effects of fire fighting that forcefully intervened by fire lines. Since the main drawback of the model validation is the general unavailability of experimental data in terms of more frequent perimeter measurements, a major goal in the comparison of model predictions to data consists of providing a general proof of concept of model adaptivity.

As the other reviewer commented, ideally, the parameters $a, b$ and $c$ should be determined from a set of experimental or field data and then be applied to other experimental or field fire scenarios. This method is also used in machine learning, where a model is trained with one set of data and then applied to another set. Indeed, the machine learning outcome is equivalent to the parameter estimation by solving an inverse problem. Yet, in both cases, the availability of sufficient data is crucial, but should not prejudice the proposal and evaluation of different methodological settings.

(a)

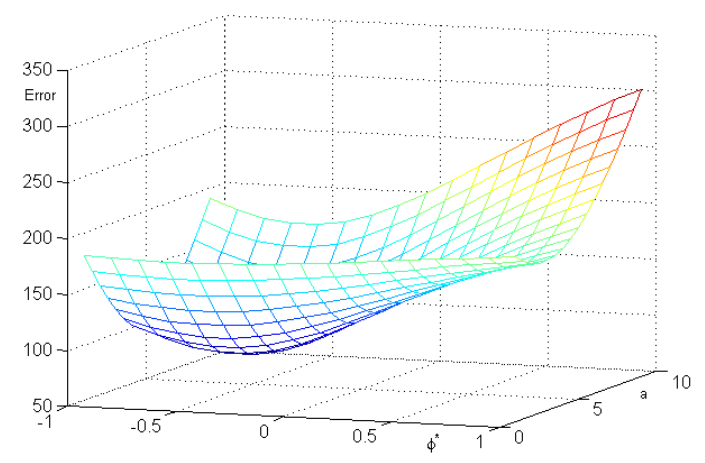

(b)

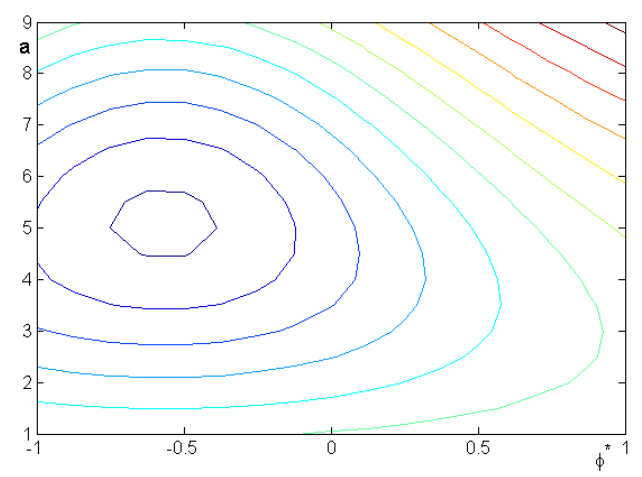

Figure 4. Biparametric cost function depending the parameter set $\left(a, \phi^{\star}\right)$. (a) Cost function. (b) Contour function.

Propagation in dependence of fuel and wind direction. Given model (2), the adjustment can be done with respect to any subset of the set of four parameters $a, b, c, \phi$. A cost function that depends on such a parameter subset, can be best visualized with one or two parameters. In Figure 4, the cost function depending on parameters $a$ and $\phi^{\star}$ is shown as a mesh plot and by contour lines. The parameter $a$ accounts for the fuel constant, and $\phi^{\star}$ corresponds to the wind direction; i.e., assuming that the wind direction is not given by external data, it can be calculated by data fitting. In the considered model scenario, it is assumed that the wind direction is stationary, i.e. does not change over time. A variation of wind direction can be formulated by an extended model, but the main obstacle are the available data that can nurture that extended model. The goal of adjusting the wind direction parameter $\phi^{\star}$ is to validate a range of representative and reasonable directions.

\section{CONCLUSION}

The presented study consisted of using experimental data to validate variants of a fire propagation front model adapting a numerical and computational solution. For this, a reference model was defined upon available standard models; this model was confronted with geospatial data of shapefile type provided by CONAF. The 
propagation of a fire was computationally simulated with Matlab by developing an inverse problem, where optimal influential parameters of the phenomenon where identified. Along with this, the sensitivity of the parameters obtained where analyzed. It is worth highlighting the relevance of using geospatial data; we used the same formats that CONAF uses in its simulations (Castillo et al. 2004).

Subsequent future work can generate guidelines for the collection of bushfire data. One idea is to use cell phone applications that allow a landowner to model and simulate a controlled burning previously in order to get a better control of its spread. An open designed modelling and simulation platform would allow a comparison of different models, including different model types and model function variants. The current system that a firefighting agency as CONAF uses to simulate real-time firefighting can be complemented and enriched by incorporating suitable geospatial data through a simulation in the Python environment on the Arcgis platform, or a file format compatible with the Wildfire tool. We conclude with the expectation and perspective that a key to the effective and safe extinction of bushfire consists of the best possible knowledge on the possibilities of how a fire might behave and spread.

\section{ACKNOWLedgement}

The first author would like to thank the support from the Corporación Kiñe Füxa Ruka Kimün and the IPCFT Lagos. The second author would like to thank the grant within the programme Doctorado en Didáctica de la Matemática, Universidad Católica del Maule. The main results of this paper where obtained while the second author studied within the master programme of applied mathematics at Universidad Católica de Temuco, Chile, under the supervision of the first author. The hospitality of the programme and the engineering faculty is gracefully acknowledged.

\section{REFERENCES}

Alexander, M. \& Cruz, M. (2013), 'Are the applications of wildland fire behaviour models getting ahead of their evaluation again?', Environmental Modelling and Software 41, 65-71.

Beer, T. (1993), 'The speed of a fire front and its dependence on wind speed', International Journal of Wildland Fire 3(4), 193-202.

Cassagne, N., Pimont, F., Dupuy, J.-L., Linn, R., Mårell, A., Oliveri, C. \& Rigolot, E. (2011), 'Using a fire propagation model to assess the efficiency of prescribed burning in reducing the fire hazard', Ecological Modelling 222(8), 1502-1514.

Castillo, M., Julio, G. \& Pedernera, P. (2004), 'Diseño e implementación de herramientas computacionales para la prevención y combate de incendios forestales', Laboratorio de Incendios Forestales, Departamento de Manejo de Recursos Forestales, Universidad de Chile .

Dumond, Y. (2009), An applied step in forest fire spread modelling, pp. 32-38.

Finney, M. (1998), 'Farsite: Fire area simulator - model development and evaluation', USDA Forest Service Research Papers RMRS (RMRS-RP-4), 1-36.

Ghisu, T., Arca, B., Pellizzaro, G. \& Duce, P. (2014), 'A level-set algorithm for simulating wildfire spread', CMES - Computer Modeling in Engineering and Sciences 102(1), 83-102.

Hilton, J., Miller, C., Sullivan, A. \& Rucinski, C. (2015), 'Effects of spatial and temporal variation in environmental conditions on simulation of wildfire spread', Environmental Modelling and Software 67, 118127. cited By 32.

Mallet, V., Keyes, D. \& Fendell, F. (2009), 'Modeling wildland fire propagation with level set methods', Computers and Mathematics with Applications 57(7), 1089-1101.

Osher, S. \& Sethian, J. (1988), 'Fronts propagating with curvature-dependent speed: Algorithms based on hamilton-jacobi formulations', Journal of Computational Physics 79(1), 12-49.

Rehm, R. G. \& McDermott, R. J. (2009), 'Fire-front propagation using the level set method', US Department of Commerce, National Institute of Standards and Technology .

Sethian, J. (1996), 'A fast marching level set method for monotonically advancing fronts', Proceedings of the National Academy of Sciences of the United States of America 93(4), 1591-1595.

Sullivan, A. (2007), 'A review of wildland fire spread modelling, 1990-present, 1: Physical and quasi-physical models', arXiv preprint arXiv:0706.3074 .

Viegas, D. (2004), 'Slope and wind effects on fire propagation', International Journal of Wildland Fire 13(2), 143-156.

Weise, D. R. \& Biging, G. S. (1994), 'Effects of wind velocity and slope on fire behavior', Fire Safety Science 4, 1041-1051. 